\title{
Optic Disc and Optic Cup Segmentation Methodology for Glaucoma Detection
}

\author{
Mohamed Aouf ${ }^{1}$, Dalia Ali $^{2}$, Ghada Kareem ${ }^{3}$ \\ ${ }^{1}$ Department of Biomedical Engineering, Higher Technological Institute, Cairo, Egypt. \\ ${ }^{2}$ Department of Electronics and Communication Engineering, Zagazig University, Cairo, Egypt. \\ ${ }^{3}$ Department of Biomedical Engineering, Higher Technological Institute, Cairo, Egypt. \\ ORCIDs: 0000-0002-7685-9132 (Mohamed Aouf) , 0000-0002-4491-6550 (Dalia Ali), 0000-0003-3954-1456 (Ghada kareem)
}

\begin{abstract}
A retinal image is an essential tool for ophthalmology diagnosis. It is necessary for the early detection of many retinal diseases including glaucoma. Glaucoma acquires a second place in the leading causes of blindness worldwide. It damages the optic nerve which is responsible for connecting 1.2 million different nerve fibers to the brain. Once the optic nerve is destroyed it can not be treated but its early diagnosis and treatment can prevent the vision loss. The detection of glaucoma is usually performed by measuring the ratio between the optic disc (OD) and the optic cup (OC). The average ratio between the $\mathrm{OD}$ and the $\mathrm{OC}$ is 0.3 . If it is higher than 0.3 , then it is an indication of glaucoma. This paper aims to present an image processing algorithm for automatic identification of glaucoma by using active contour (snake) model to contour the OD and the OC boundaries that will help calculate the cup to disk ratio (CDR). The proposed model will help ophthalmologists to monitor any changes occur in the optic nerve.
\end{abstract}

Keywords - Active Contour Model, Image Processing, Glaucoma, Retinal Images, Snake Model.

\section{INTRODUCTION}

Glaucoma is a result of sudden increases in the Intra-ocular pressure (IOP) of the eye [1]. The IOP is responsible for the maintenance and protection of the eye from deformation. The increase in the IOP leads to damage the optic nerve and gradually increases in the blind spots diameter which leads to vision loss, as shown in Figure 1.

Many difficulties are commonly related to image processing algorithms applied for glaucoma detection that make it hard to achieve high accuracy performance with the diagnosis done by ophthalmologist experts. Some common problems are mentioned below:

- Some noise may be found in the fundus images during image acquisition that leads to false detection of the target object.
- Some bright artifacts and dark artifacts around the OD boundaries can mislead the accurate segmentation of the OD boundaries.

- Some retinal noise such as exudates which has similar intensity and color as the OD.

- The blood vessels that interfere with the OC make it hard to accurately estimate the OC boundaries.

- Some reflections, illuminations, and low contrast that are found in some dataset.

Accurate glaucoma diagnosis can be obtained by three methods. First, apply the air puff exam using tonometry [2] to calculate the IOP. Second, examine the level of damage in the optic nerve. Third, check the visual field. Since the IOP in both low-tension glaucoma and elevating glaucoma is high and testing the visual field requires special equipment. Therefore, the most proper technique to evaluate glaucoma is to examine the optic nerve. The optic nerve is examined by calculating the cup to disc ratio (CDR) which is the ratio between the area of the disc to the area of the cup [3]. Calculating the CDR ratio is very expensive and performed only by experts so it will be more practical to apply an automatic image processing algorithm to calculate CDR.

\section{RELATED WORK}

Glaucoma diagnostic is based on time consuming manual observations therefore developing an image processing algorithm will help the ophthalmologists to decrease cost and effective time manner. The following reviewed articles used different image processing algorithms for detecting glaucoma.

Fraga et al. [4] proposed an algorithm for OD segmentation by applying hough transform and fuzzy convergence. First, the OD was localized before applying hough transform for OD segmentation. For vascular tree segmentation canny filter was applied then computes the histogram of the region of interest (ROI).

Mishra et al. [5] used active contour for obtaining CDR. In this method, the green channel was extracted and morphological operations were applied for illumination 


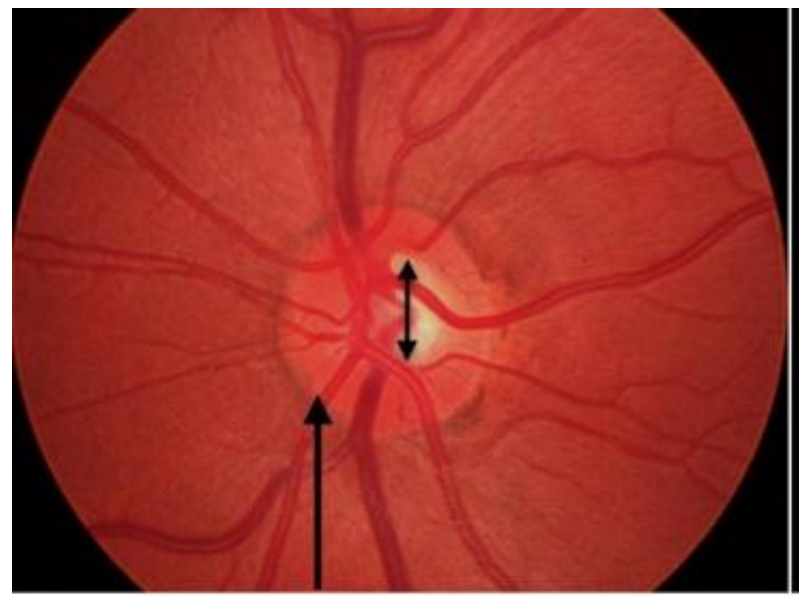

Normal optic nerve head

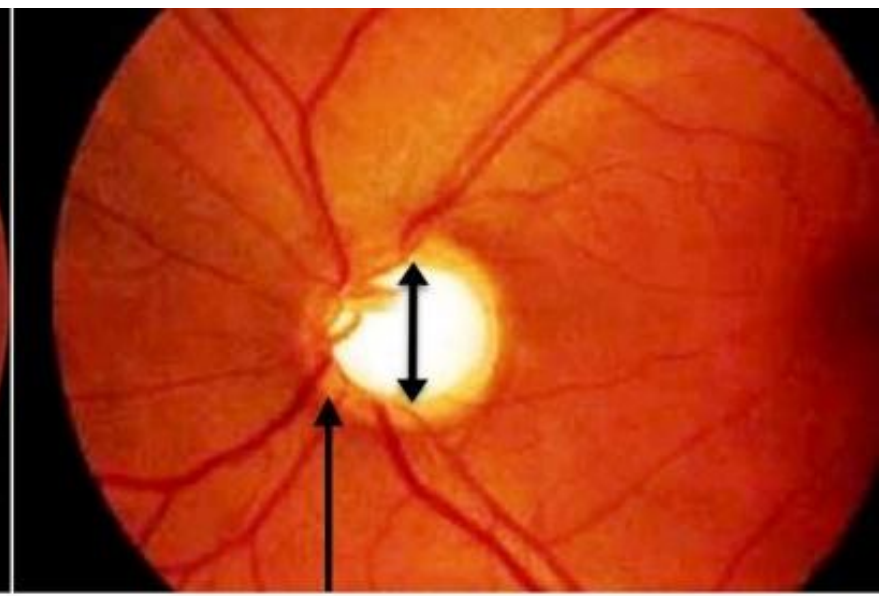

Glaucomatous cupping

Fig. 1. (a) Normal retinal image, (b) Optic nerve cupping.

correction. The blood vessels were removed and the optic nerve head was normalized. Finally, CDR was calculated after using multi thresholding and active contour to detect the boundaries of both OC and OD.

Tjandrasa et al. [6] applied active contour and hough transform for optic disc extraction. First, enhance the retinal image and used holomorphic filtering for correcting illumination. This filter works in two ways. First, a gaussian low pass filter was applied. Second, obtaining the edge of the filtered by using dilation to remove the blood vessel. Then, the optic disc was detected using hough transform. Finally, the active contour was used with specific termed selective binary and gaussian filtering regularized level set.

Chandra and Nirmala et al. [7] used wavelet and k-mean to obtain CDR. First, ROI was identified then apply threshold for every $k$ centered, each cluster was defined. Each point was associated with the closer centroid to make a group of data. Then it was recalculated to other centroids like boundaries of the cluster to be used in the next tread. A loop was used to route the place of the centroids until it was not moving to decrease the squared error function. The $\mathrm{k}$ means conducted on the mask of the ROI then the image was segmented using Gabor wavelet transform. Finally, some morphological feature was applied and CDR was calculated.

Yin et al. [8] used model-based segmentation. These algorithms combine both optimal channel selection of the OC, OD, and hough transform. First, the image was enhanced and determines the best model to process based on the image contrast ratio. Then, used circular hough along with a canny edge to detect the OD boundary. A direct fitting ellipse approach was used to smooth the boundary. Optic cup segmented, local entropy, and thresholding were applied. Then, extracting the OC. Finally, the CDR was calculated.
Ishraq et al. [9] used Gabor wavelet for glaucoma detection. First, preprocessing the image by removing blood vessels. Then, the OD was segmented using compositing vessel information and the boundary detection of the OD varies along with the line profile.

Bokhari et al. [10] used Fourier transform, active contour, and circular hough transform for OD and OC detection. First, the image was normalization and enhancement. Then, extract the ROI of interest. The OD was segmented using active contour and hough transform. Finally, the OC was segmented to calculate CDR and differentiate between healthy and unhealthy images.

Aouf et al. [11] used the wavelet transform to extract features for glaucoma. The image was enhanced using gabor filter. A discrete wavelet along with teager-kiaser operator was applied to extract the features. Fuzzy c-mean clustering (FCM) was used to classify the degree of glaucoma.

Abdullah et al. [12] the OD was segmented using an active contour model based on FCM clustering. Morphological operations were applied to enhance and normalize the image. FCM clustering was applied to locate the OD center. The active contour model was applied to locate the boundary of the OD.

\section{METHODOLOGY}

Segmentation is a section in image processing which means separation of the desired information or a target area of interest for further processing. An area of interest means a group of pixels defined with boundary which takes different shapes as ellipse or irregular and circular shape. The segmentation process does not provide information about all images but only pixel data of the desired area of interest. 
The image segmentation process can be done in different ways depending on the similarity and discontinuity of the intensity values. Thresholding, split region, boundary detection and active contour model are examples of image processing segmentation algorithms.

Active contour model [13] is an algorithm for segmentation using the energy restraint of the image to separate certain regions of interest. It is applied to various medical applications as computerized tomography, Magnetic Resonance Imaging, fundus images, and cardiac image. It is also used in stereo and motion tracking. The main application of the active contour model is to define a shape in the image and form a closed contour for the point of interest.

For the part of interest illustrated in the image, the contour is explained depending on the force and constraint in the desired region [14]. There are different types for the active model such as geometric model, gradient model, snake model, and balloon model.

Snake is a model that solves a big function of the segmentation instance. This model works to identify and to outlines the desired area required for segmentation. It needs a defined knowledge about the desired area contour, usually for a complex entity. This model is configured by utilizing spline to decrease the energy due to many forces governing the image.

The snake model is mathematically presented as a group of polynomials to utilize the geometric figure, such as curves. The spline of decreasing energy presents the constraint forces and pulled by the help of both inner and outer forces of the image depend on suitable contour features. The snake model ratifies the deformation model to the desired image through the energy minimization. The snake model usually applies cubic polynomial between the higher-order polynomial could be formed, but it is avoided as a result of many unneeded local properties to deal with.

The snake model works perfectly with a complex object by dividing the target image into very small targets. It is utilized to be variable in its shape and location while tending to search through the minimal energy state. Snakes propagate through the images domains to decrease the energy function and intend to dynamically move to the local minimum. Snake is mathematically represented in (1):

$$
V(s, t)=x(s, t), y(s, t)
$$

$\mathrm{x}, \mathrm{y}$ : represents the two-dimensional coordinates of the curve.

$\mathrm{v}$ : represent spline criteria and it ranges from 0 to 1.

$\mathrm{s}$ : represent linear criteria belong to $\{0,1\}$. t: represent the time criteria belong to $\{0$, infinity $\}$.

The sum of all energy is equal to: (i) The internal energy $\left(E_{i}\right)$ which depends on the spline degree related to the target image shape, (ii) the external energy $\left(E_{o}\right)$ which represents the outer force gained by the user and many other energy factors, (iii) $\mathrm{E}_{\mathrm{I}}$ which represents the image energy under consideration, which mathematically represented in (2):

$$
\mathbf{E}_{\mathbf{T}}=\mathbf{E}_{\mathbf{i}}+\mathbf{E}_{\mathbf{o}}+\mathbf{E}_{\mathbf{I}}
$$

The inner energy represents a hybrid goal in smoothness desired contour object, which mathematically represented in (3):

$$
E_{i}=\delta\left|\frac{\sigma v}{\sigma s}\right|^{2}+\beta\left|\frac{\sigma^{2} v}{\sigma s^{2}}\right|
$$

$\sigma$ : represent how long the snake will be expanded and also represent its elasticity.

$\beta$ : represent the snake level of rigidity.

The outer energy which mathematically represented in (4):

$$
E_{o}=W_{1} I(x, y)+W_{2}|\nabla I(x, y)|^{2}+\cdots
$$

It describes the snake near the desired local minimum. It also represents using high-level interactions.

$\mathrm{W}_{1}$ : represent the line efficiently.

$\mathrm{W}_{2}$ : represent the edge efficiently.

According to the $\mathrm{W}_{1}$ and $\mathrm{W}_{2}$ values, the model will adapt itself to the darker pixel area when it is a positive value and move to the bright pixel when it is a negative value.

In this section active (snake) model will be applied for detecting the OD and OC boundaries. The contoured OD and OC will be used to calculate CDR that will help to detect glaucoma. Before applying the snake model the retinal image is converted to hue, saturation, Lightness (HSL) color space. The blood vessels are eliminated using morphological operations. Finally, the snake model is applied to contour the OD and OC. The block diagram of the proposed algorithm is shown in Figure 2. The preprocessing steps and the snake model are discussed in detail in the following subsection.

\section{- H-S-L Color Space}

HSL is an abbreviation for (hue, saturation, Lightness). It is useful to separate the image intensity from the rest of the color information unlike RGB color space as shown in Figure 3. The HSL color space is useful while plotting an image histogram. 


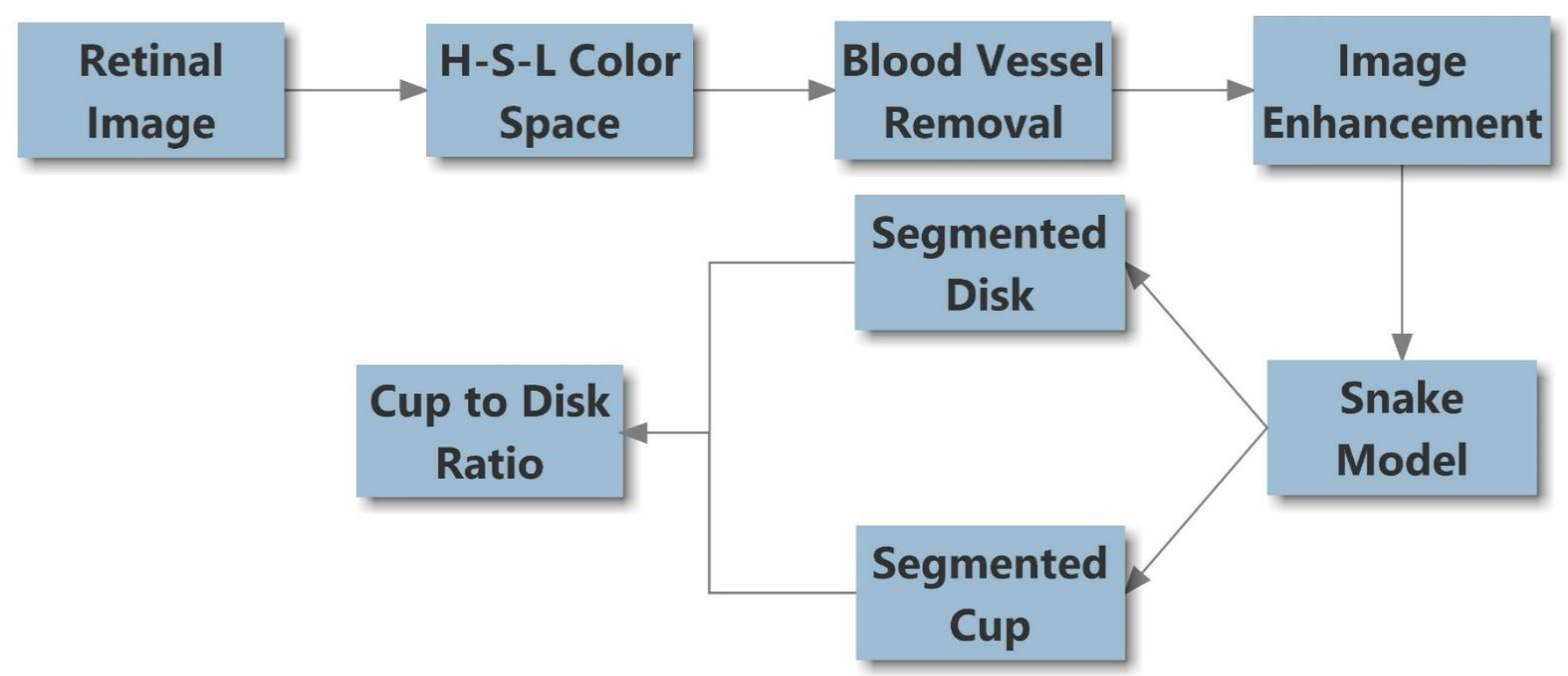

Fig. 2. Flow chart of the proposed algorithm.

\section{- Blood Vessel Removal}

The blood vessel is removed using morphological closing which will help to preserve the foreground while eliminating any other region in the image. Morphological closing is used to remove the unnecessary blood vessel components. A median filter is then applied to specify the edges and to make the image even smoother, as shown in Figure 4.
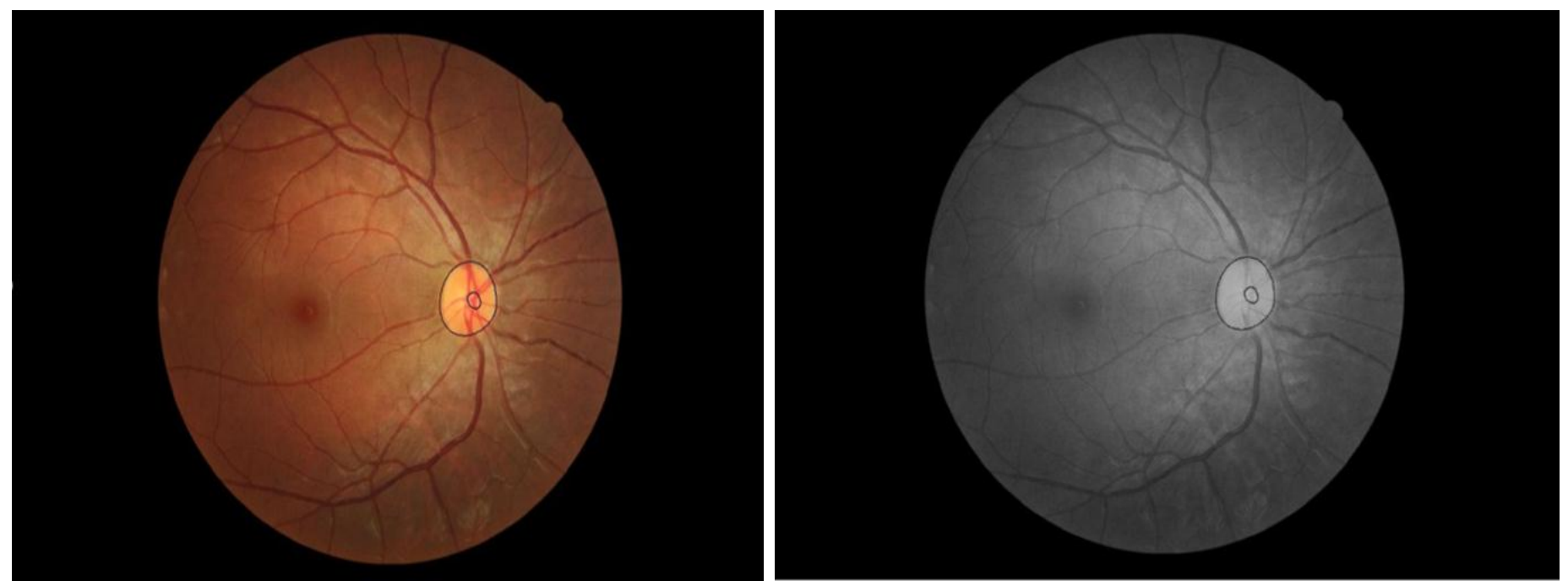

(a)

(b)

Fig. 3. (a) The colored retinal image, (b) H-S-L color space. 


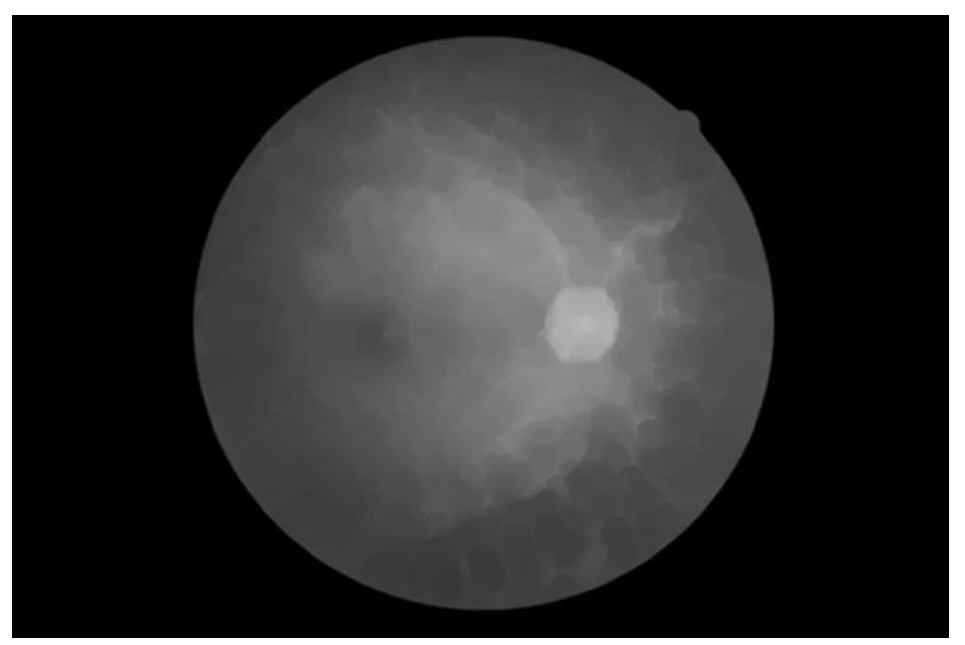

(a)

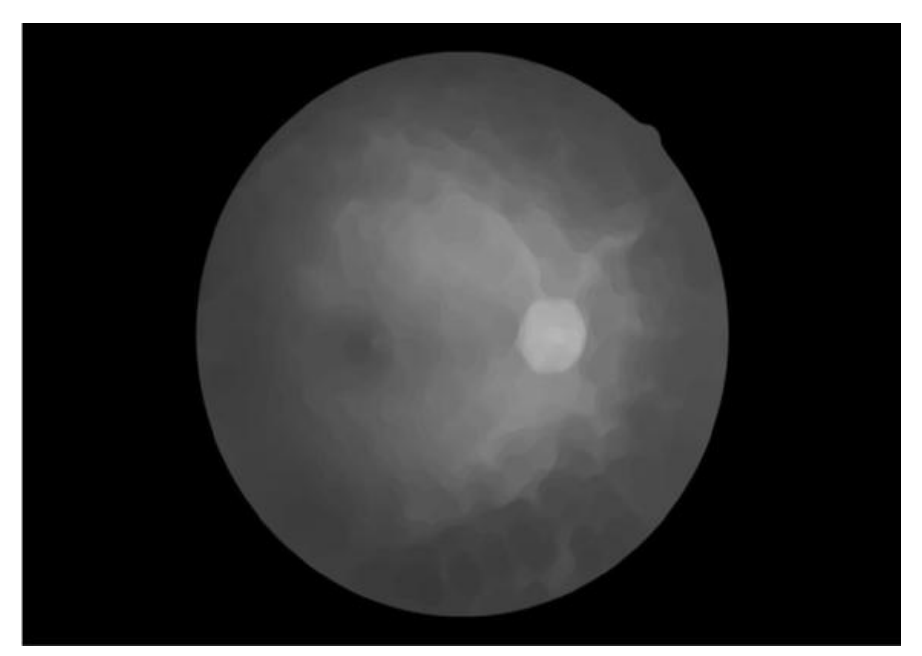

(b)

Fig. 4. (a) Blood vessel removal, (b) Enhanced image after median filter.

\section{- Detecting the Center of the Optic Cup}

The center of the optic cup is detected by using the image histogram. An image histogram is a graph plotting representing different color intensities in the image. It displays how many pixels of every possible color is in the image, as shown in Figure 5 (a). Every bar on the image histogram represents one intensity level. The brightest area in the image is the optic cup. A for loop is applied to specify the brightest pixel on the image using information from the histogram plot and used to get the initial point for active contour, as shown in Figure 5 (b).

\section{- Applying Snake Model}

Histogram

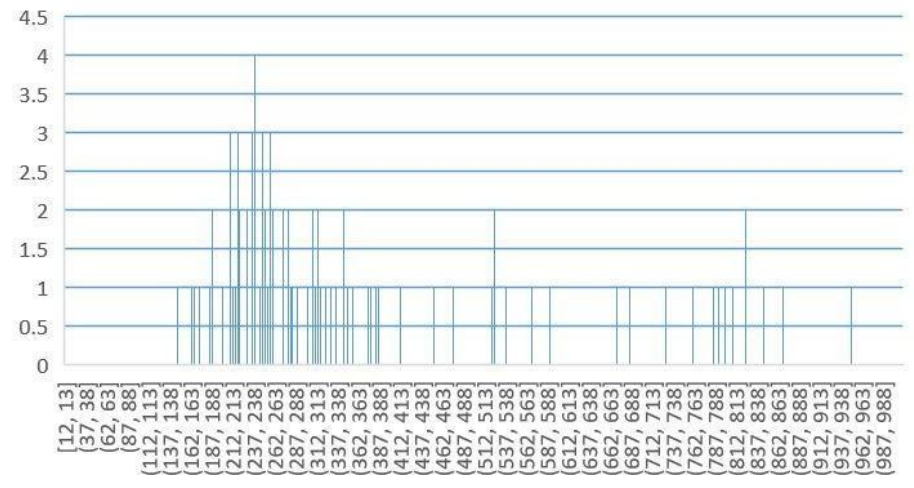

(a)
A snake is an active (moving) contour, in which the points are attracted by edges and other boundaries. According to (3) and (4), the parameters are specified by the followed values:

Sigma: Sigma used to calculate image derivatives, default 10.

Wline: Attraction to lines, default 0.04 .

Wedge: Attraction to edges, default 2.0.

Finally, the result of the snake model is plotted on the RGB image (main image), as shown in Figure 6. The result is plotted on the RGB image as it is essential to calculate the performance of the retinal image analysis methods by

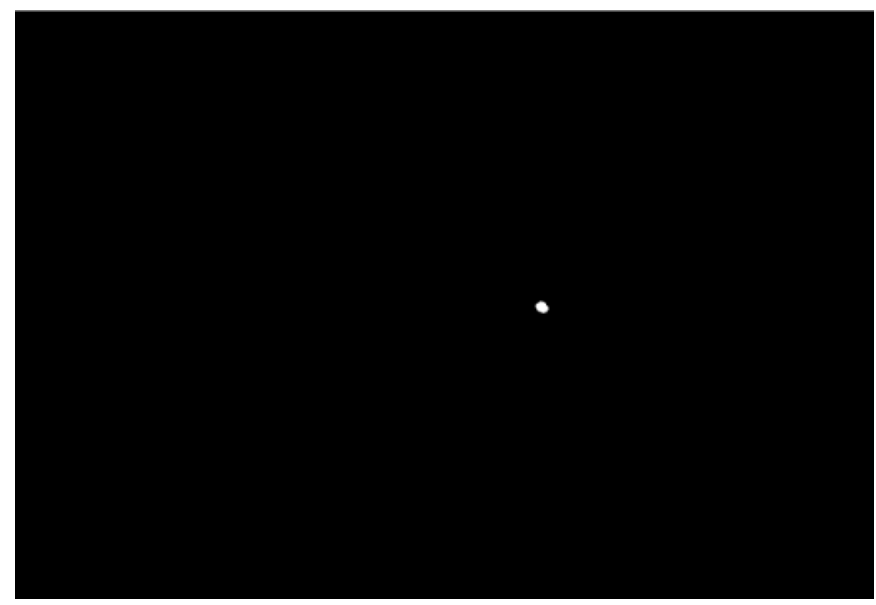

(b)

Fig. 5. (a) Histogram plot, (b) Optic cup center. 
measuring the agreement level between the output and the annotation image marked by ophthalmologist experts. After applying the snake model, the CDR is calculated.

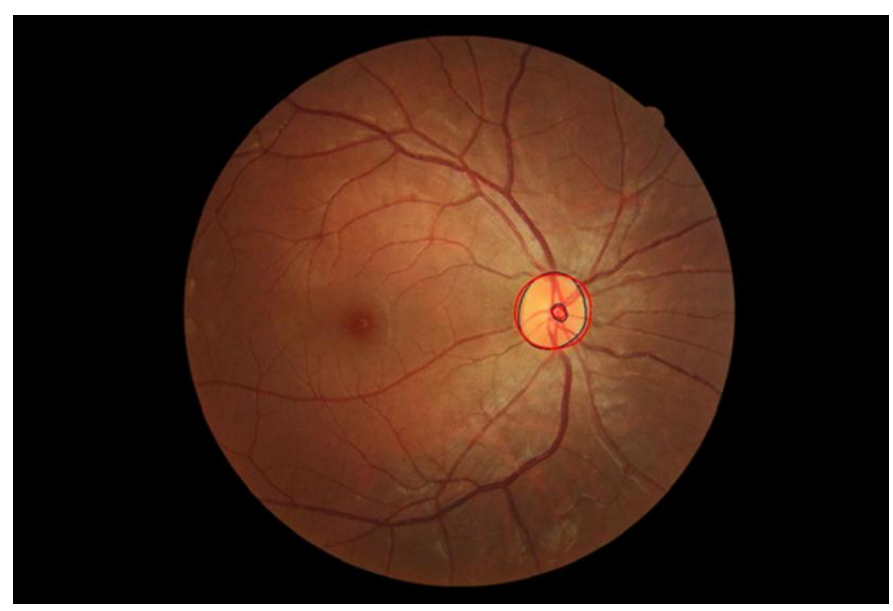

Fig. 6. Active (snake) model contouring result.

\section{RESULTS AND DISCUSSION}

The model is performed using MATLAB 2018a environment with intel core i7- $2.5 \mathrm{GHz}$ processor. The colored retinal image is first converted to HSL color space. Then, the blood vessels are removed using morphological closing and enhancing the image using a median filter. The information from the histogram is used to detect the optic cup center. Finally, the snake model is applied to contour the OD and the OC boundaries, as shown in Figure 7.

The dataset used for glaucoma detection called RIGA dataset. It is an abbreviation for the retinal fundus image for glaucoma analysis [15]. The images of this dataset are divided into different groups. One of the groups called Bin-Rushed which consists of 195 retinal images. Each image is marked by six different ophthalmologist experts. The images of this dataset are saved in JPG and TIF format with resolution $2376^{*} 1584$. The performance of the snake model is compared to the contouring done by six different ophthalmologist experts.

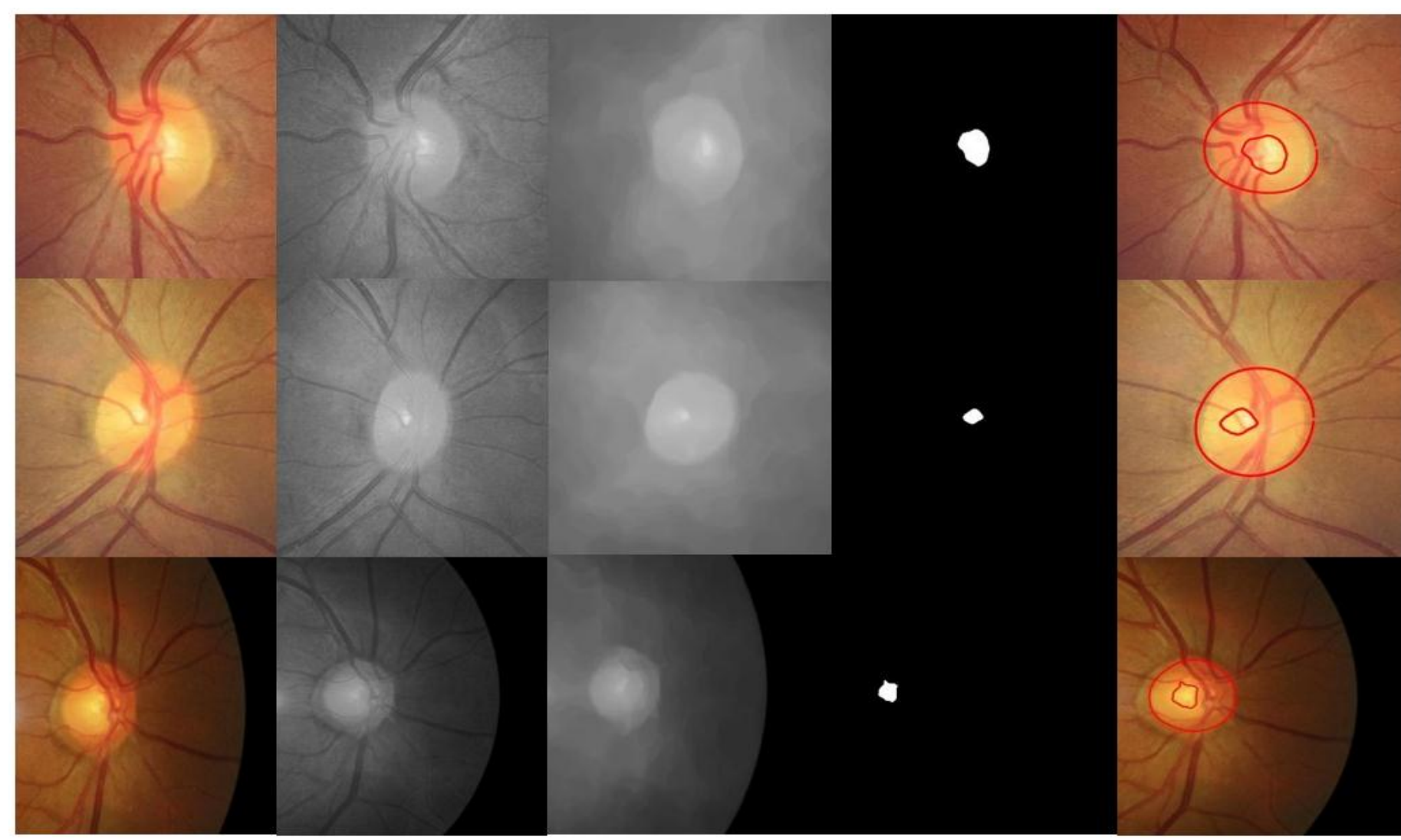

(a)

(b)

(c)

(d)

(e)

Fig. 7. (a) Main image, (b) H-S-L color space, (c) After morphological closing and median filter, (d) Optic cup center, (e) Final result of the snake model. 
The accuracy achieved compared to the six experts results is shown in Figure 8. The algorithm is tested using 195 retinal images. A total of 31 images are eliminated due to failure in detecting neither the OD boundaries or OC boundaries due to the low resolution of the images. A total of 164 images are used for testing. Based on the data illustrated in Table 1, ophthalmologists numbers 3 and 6 achieves the highest performance using 151 and 144 retinal images. The ophthalmologist number 1 achieves the least level of agreement with the proposed algorithm with accuracy $79 \%$ (lack of agreement between the result and the annotation images). Figure 9 shows retinal images the snake failed to contour the OC or the OD. Figure 10 shows the retinal images the active snake model did not correctly contour the OC or the OD. Figure 11 shows the retinal images that the active snake model succeeded to contour the OD and the OC boundaries.

\section{Accuracy}

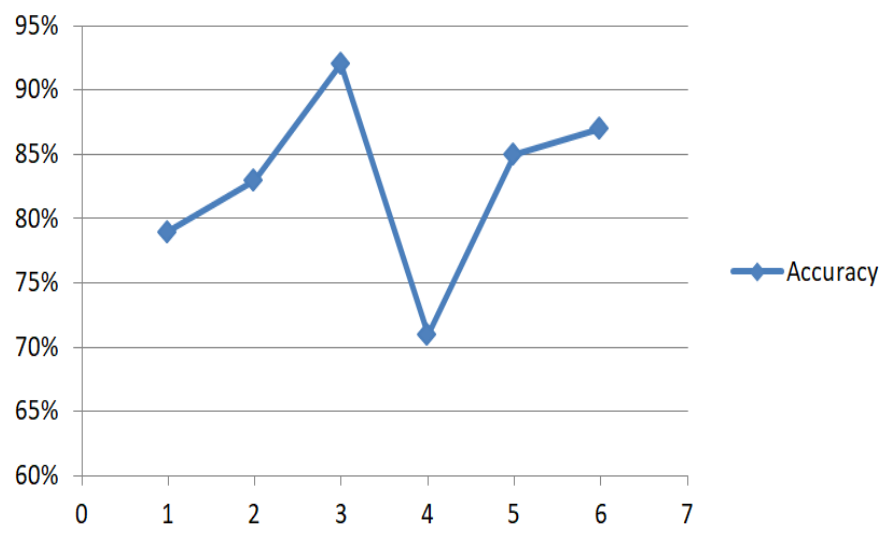

Fig. 8. The accuracy result of the six ophthalmologists results for Bin-rushed dataset images.

Table 1. The results compared to the ophthalmologist experts annotation images.

\begin{tabular}{|c|c|c|c|c|c|c|}
\hline & Ophth.1 & Ophth.2 & Ophth.3 & Ophth.4 & Ophth.5 & Ophth.6 \\
\hline \multicolumn{7}{|c|}{ Total no. of images 195} \\
\hline $\begin{array}{l}\text { No. of images not } \\
\text { located }\end{array}$ & 31 & 31 & 31 & 31 & 31 & 31 \\
\hline No. of test images & 164 & 164 & 164 & 164 & 164 & 164 \\
\hline Level of agreement & 130 & 138 & 151 & 118 & 140 & 144 \\
\hline Accuracy & $79 \%$ & $83 \%$ & $92 \%$ & $71 \%$ & $85 \%$ & $87 \%$ \\
\hline
\end{tabular}

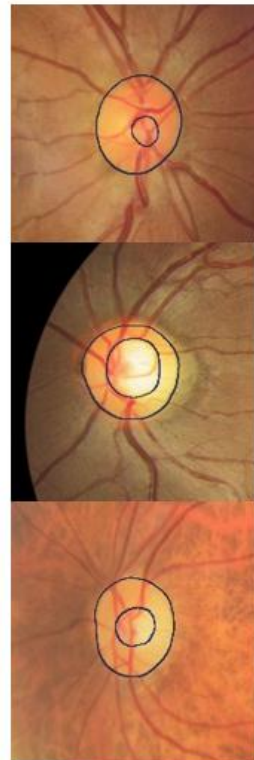

(a)

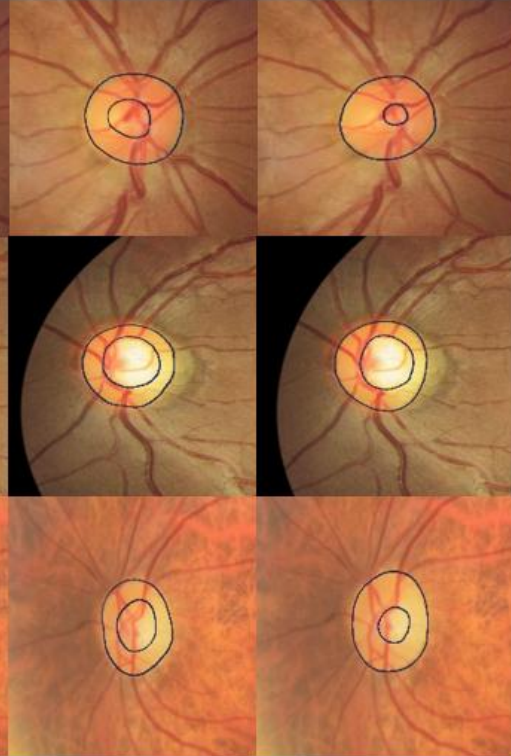

(b) (c)

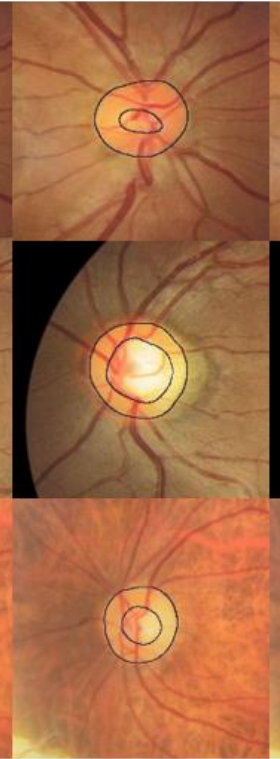

(d)

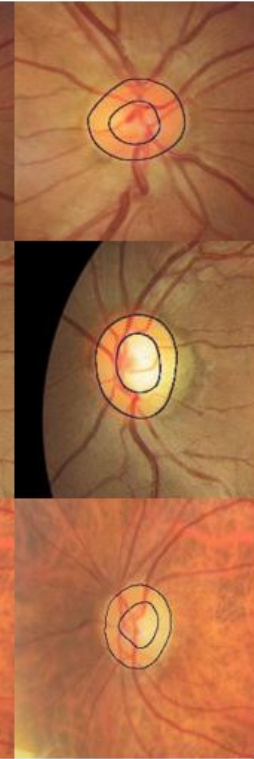

(e)

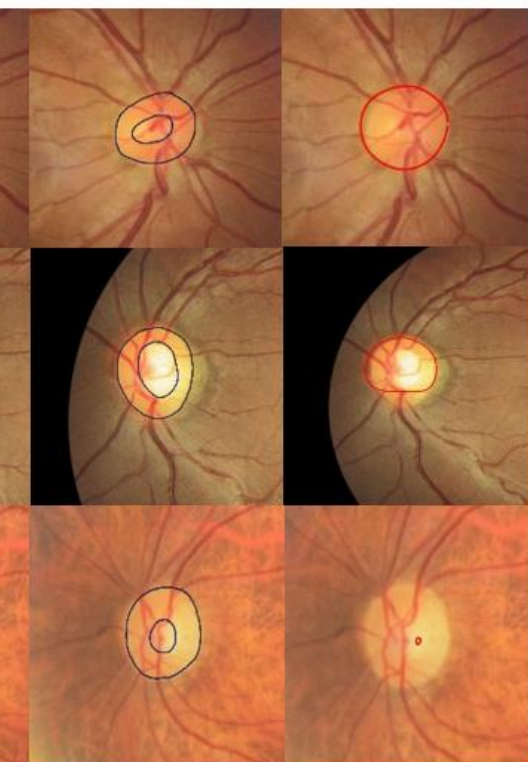

(f) (g)

Fig. 9. Results of images failed to contour OC or the OD: (a) Ophth.1, (b) Ophth.2, (c) Ophth.3, (d) Ophth.4, (e) Ophth.5, (f) Ophth.6, (g) Proposed algorithm. 


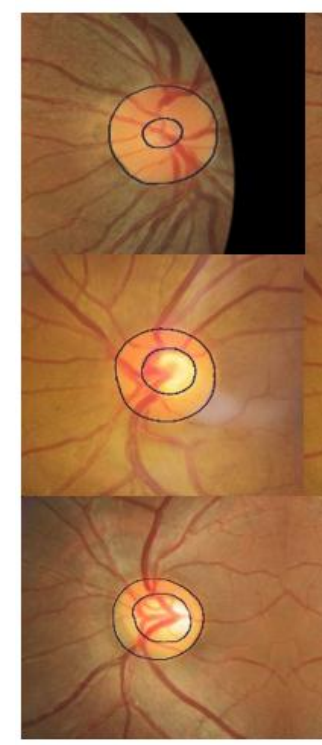

(a)

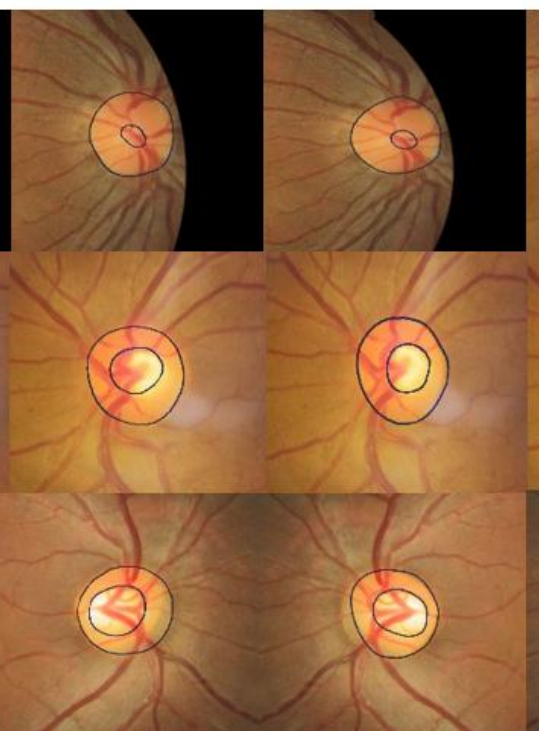

(b)

(c)

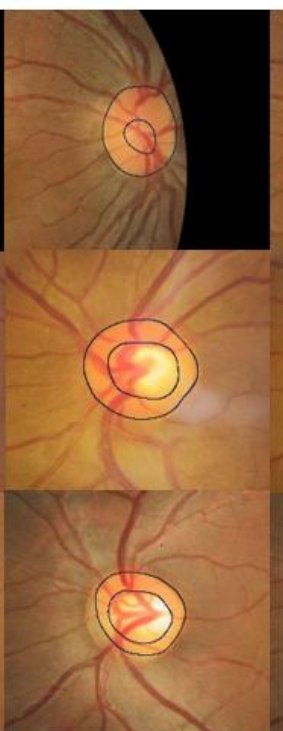

(d)

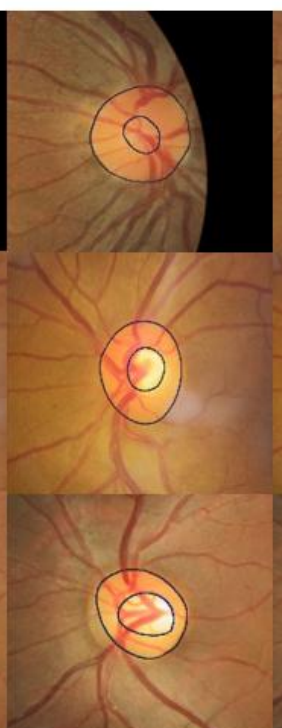

(e)

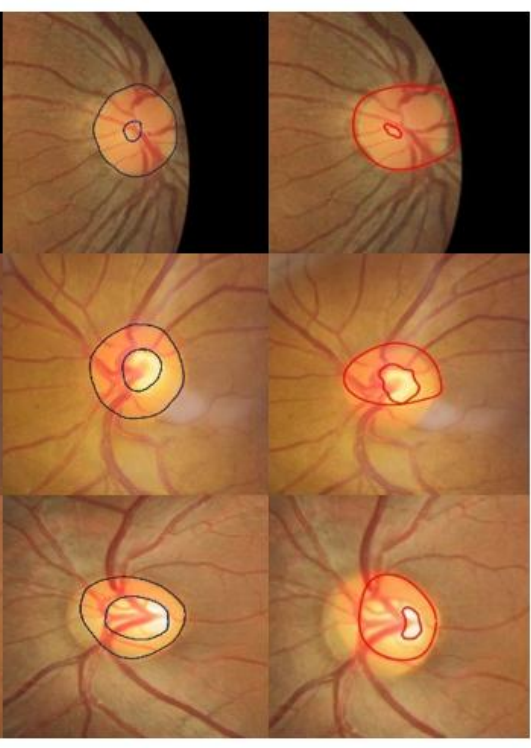

(f)

(g)

Fig. 10. Results of images the model did not correctly contour the OC or the OD: (a) Ophth.1, (b) Ophth.2, (c) Ophth.3, (d) Ophth.4, (e) Ophth.5, (f) Ophth.6, (g) Proposed algorithm.

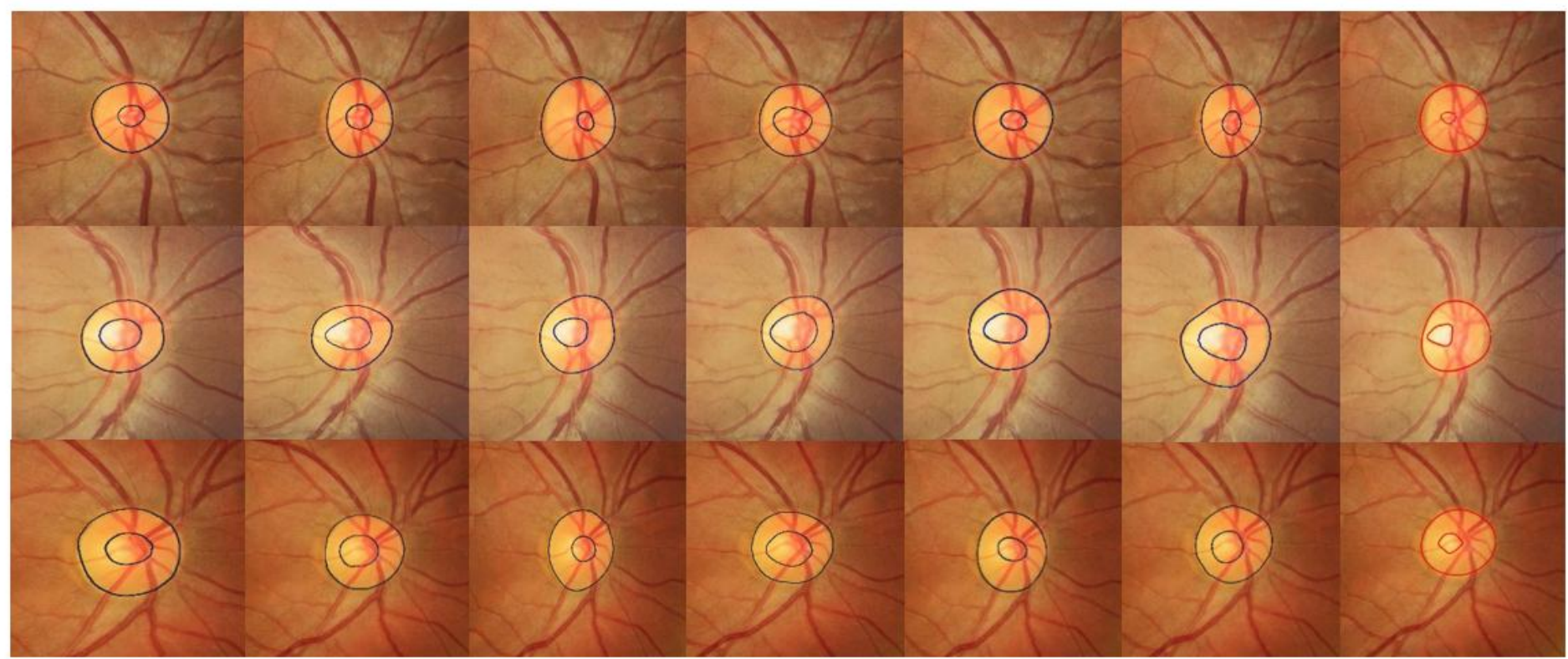

(a)

(b)

(c)

(d)

(e)

(f)

(g)

Fig.11. Results of images are correctly contour the OC and the OD: (a) Ophth.1, (b) Ophth.2, (c) Ophth.3, (d) Ophth.4, (e) Ophth.5, (f) Ophth.6, (g) Proposed algorithm.

\section{CONCLUSIONS}

This paper presented an image processing algorithm for detecting glaucoma which is one of the chronic retinal diseases that destroys the optic nerve responsible for transferring photoreceptors to the brain. For glaucoma detection, active contour (snake) model was applied to contour OD and OC to be able to calculate the CDR. The result of the algorithm was compared to annotation images marked by six different ophthalmologist experts to avoid the error of any other algorithm. The proposed algorithm processed effectively and can be utilized as a helpful tool in glaucoma detection. 


\section{REFERENCES}

[1] Haleem, M.S., Han, L., Van Hemert, J. and Li, B., 2013. Automatic extraction of retinal features from colour retinal images for glaucoma diagnosis: a review. Computerized medical imaging and graphics, 37(7-8), pp.581-596.

[2] Thakur, N. and Juneja, M., 2018. Survey on segmentation and classification approaches of optic cup and optic disc for diagnosis of glaucoma. Biomedical Signal Processing and Control, 42, pp.162-189.

[3] Almazroa, A., Burman, R., Raahemifar, K. and Lakshminarayanan, V., 2015. Optic disc and optic cup segmentation methodologies for glaucoma image detection: a survey. Journal of ophthalmology, 2015.

[4] Fraga, A., Barreira, N., Ortega, M., Penedo, M.G. and Carreira, M.J., 2011, February. Precise segmentation of the optic disc in retinal fundus images. In International Conference on Computer Aided Systems Theory (pp. 584591). Springer, Berlin, Heidelberg.

[5] Mishra, M., Nath, M.K. and Dandapat, S., 2011. Glaucoma detection from color fundus images. International Journal of Computer \& Communication Technology (IJCCT), 2(6), pp.7-10.

[6] Tjandrasa, H., Wijayanti, A. and Suciati, N., 2012. Optic nerve head segmentation using hough transform and active contours. Telkomnika, 10(3), p.531.

[7] Chandrika, S. and Nirmala, K., 2013. Analysis of CDR detection for glaucoma diagnosis. International Journal of Engineering Research and Application, 2(4), pp.23-27.

[8] Yin, F., Liu, J., Wong, D.W.K., Tan, N.M., Cheung, C., Baskaran, M., Aung, T. and Wong, T.Y., 2012, June. Automated segmentation of optic disc and optic cup in fundus images for glaucoma diagnosis. In 2012 25th IEEE international symposium on computer-based medical systems (CBMS) (pp. 1-6). IEEE.

[9] Irshad, S., Yin, X., Li, L.Q. and Salman, U., 2016, December. Automatic Optic disc Segmentation in Presence of Disk Blurring. In International Symposium on Visual Computing (pp. 13-23).

[10]Bokhari, F., Syedia, T., Sharif, M., Yasmin, M. and Fernandes, S.L., 2018. Fundus image segmentation and feature extraction for the detection of glaucoma: A new approach. Current Medical Imaging Reviews, 14(1), pp.7787.

[11] Aouf, M. and Kareem, G., 2018. Glaucoma Diagnosis FCM_Tk Algorithm Based on Funds Camera. International Journal of Intelligent Computing and Information Sciences, 18(2), pp.29-38.
[12] Abdullah, A.S., Rahebi, J., Özok, Y.E. and Aljanabi, M., 2019. A new and effective method for human retina optic disc segmentation with fuzzy clustering method based on active contour model. Medical \& biological engineering \& computing, pp.1-13.

[13] Hemalatha, R.J., Thamizhvani, T.R., Dhivya, A.J.A., Joseph, J.E., Babu, B. and Chandrasekaran, R., 2018. Active Contour Based Segmentation Techniques for Medical Image Analysis. Medical and Biological Image Analysis, p.17.

[14] Kroon, D. J. (2010). Active shape model (ASM) and active appearance model (AAM). MATLAB implementation, www:

http://www.mathworks.com/matlabcentral/fileexchange/267 06-active-shape-model-asm-and-active-appearance-modelaam, 8, 22. (Access Data 8 August 2018).

[15] Almazroa, A., Alodhayb, S., Osman, E., Ramadan, E., Hummadi, M., Dlaim, M., Alkatee, M., Raahemifar, K. and Lakshminarayanan, V., 2018, March. Retinal fundus images for glaucoma analysis: the RIGA dataset. In Medical Imaging 2018: Imaging Informatics for Healthcare, Research, and Applications (Vol. 10579, p. 105790B). International Society for Optics and Photonics. 